\title{
Microgravity might affect Peri-implant Mucosal Epithelial Cells during Space Flight
}

\author{
${ }^{1}$ Akiko Tamura, ${ }^{2}$ Chihiro Masaki, ${ }^{3}$ Yoshinori Seo, ${ }^{4}$ Chisato Mukai, ${ }^{5}$ Taro Mukaibo \\ ${ }^{6}$ Yusuke Kondo, ${ }^{7}$ Tetsuji Nakamoto, ${ }^{8}$ Ryuji Hosokawa
}

\begin{abstract}
Purpose: The effect of microgravity on gingival epithelial cells (GE1) is unknown; thus, we analyzed cell proliferation as well as the gene expression patterns in GE1 cells cultured under simulated microgravity.
\end{abstract}

Materials and methods: Gingival epithelial cells were seeded and cultured at $10^{-3} \mathrm{G}$ in a three-dimensional clinostat to simulate microgravity (group CL) or in normal gravity (group C) for 10 days. Cell proliferation was analyzed by counting the numbers of cells. Real-time polymerase chain reaction was performed to amplify the krt 5, krt 13 and involucrin genes. Additionally, total protein was immunoblotted with anti-krt 13 antibody. Statistical analysis $(n=9$, three groups repeated three times) was performed (ANOVA, Tukey's test, $p<0.05$ ).

Results: Cell proliferation was significantly upregulated under microgravity based on the average number of cells. Cell proliferation and differentiation marker expression was significantly increased after culture under simulated microgravity. Western blotting showed intense krt 13 staining under simulated microgravity. The simulated microgravity environment had an accelerating effect on GE1 proliferation and differentiation.

Conclusion: These findings suggest that GE1 cells would be affected by the microgravity environment during space flight. Moreover, these findings also suggest that we could promote regeneration of gingival cells using of a simulated microgravity environment.

Keywords: Microgravity, Epithelial cell, Gene expression, Oral mucosa.

How to cite this article: Tamura A, Masaki C, Seo Y, Mukai C, Mukaibo T, Kondo Y, Nakamoto T, Hosokawa R. Microgravity might affect Peri-implant Mucosal Epithelial Cells during Space Flight. Int J Prosthodont Restor Dent 2015;5(1):10-16.

Source of support: Nil

Conflict of interest: None

\footnotetext{
${ }^{1}$ Graduate Student, ${ }^{2}$ Associate Professor

${ }^{3-6}$ Assistant Professor, ${ }^{7}$ Professor, ${ }^{8}$ Dean

${ }^{1-6,8}$ Department of Oral Reconstruction and Rehabilitation Kyushu Dental University, Fukuoka, Japan

${ }^{7}$ Department of Prosthodontics, Matsumoto Dental University Nagano, Japan
}

Corresponding Author: Ryuji Hosokawa, Dean, Department of Oral Reconstruction and Rehabilitation, Kyushu Dental University Fukuoka, Japan, Phone: 819033268988, e-mail: hosokawa@ kyu-dent.ac.jp

\section{INTRODUCTION}

Studies of astronauts and experimental animals have demonstrated that some organs and systems undergo physiological changes during and after space flight as a consequence of microgravity. The effects of microgravity have been closely associated with bone loss, muscle atrophy and decreased immune function in astronauts. ${ }^{1-3}$ An understanding of the genetic and cellular activity changes it causes is essential for a better understanding of the problems that result from exposure to microgravity, and for the development of potential countermeasures. Microgravity has been found to influence major cellular events, such as the cell cycle, cell proliferation and cell differentiation. ${ }^{4-6}$

Microgravity conditions can be produced either by space flight or free fall. Today, experimental laboratories are accessible on the International Space Station. However, laboratory availability is limited, and the duration of microgravity conditions produced by free fall is usually too short to alter cell growth and differentiation. As a result, the desire for simulated microgravity conditions on earth has led to several innovations. The clinostat, a device originating from an instrument developed to investigate gravitropism in plants, even before man had the opportunity to go to space, is an original form of the most commonly used devices today. The Rotary Cell Culture System (RCCS) developed by NASA, is a horizontally rotating cylindrical culture vessel, which offers an optimized suspension cell culture. Cells are seeded on microcarrier-bead scaffolds and, when grown in the RCCS, the cells undergo continuous free-fall conditions. This system is suitable for three-dimensional (3D) cell culture and tissue engineering. ${ }^{7,8}$ The $3 \mathrm{D}$ clinostat or the random positioning machine (RPM) is another microgravity simulation device in current use. Its basic structure is similar to the clinostat, but with two independently rotating frames, and an electromotor on each frame, controlled by dedicated software run on a computer to disperse the gravity vector throughout the whole steric angle. The mathematical algorithms used to run the electromotor vary by device, and the 3D clinostat Gravite (Kitagawa Iron Works Co, Ltd, Hiroshima, Japan) is programed to simulate a $10^{-3} \mathrm{G}$ gravitational state. 
The gingival tissue surrounding dental implants has been demonstrated to be different from that surrounding a natural tooth. The peri-implant tissue has a higher fiber content with a lower cellular content than the gingival tissue surrounding a tooth. In addition, the collagen fibers run parallel to the titanium implant surface, in contrast to gingival tissue surrounding a natural tooth, where collagen fibers penetrate the cementum and bone, and run perpendicular to the tooth surface. ${ }^{9,10}$ Thus, numerous studies have aimed to maintain healthy soft tissue around dental implants, ${ }^{11,12}$ and some cellular studies have investigated the attachment of gingival epithelial cells (GE1) to titanium surfaces ${ }^{13}$ or used tissue engineering techniques to create an oral mucosal model with adherence to titanium. ${ }^{14}$ However, the influences of microgravity on peri-implant soft tissue have not yet been clarified.

In this study, we analyzed the expression of genes related to the proliferation and differentiation of GE1 cells cultured in simulated microgravity using a 3D clinostat to investigate the effects of microgravity on gingival tissue.

\section{MATERIALS AND METHODS}

\section{Gravite (3D Clinostat, Gravity Controller)}

Gravite is a multidirectional G-force generator that cancels the cumulative gravity vector in the center of the device by simultaneously controlling the rotation of two axes, producing an environment with an average of $10^{-3} \mathrm{G}$ over time. This is accomplished by the rotation of a chamber at the center of the device to disperse the gravity vector uniformly within a spherical volume at a constant angular velocity ${ }^{15}$ (Fig. 1).

\section{Cell Culture}

The GE1 cell line, originating from the gingival epithelial tissue of temperature-sensitive SV40 large T-antigen gene transgenic C57BL/6 mice, was purchased from RIKEN BRC CELL BANK (Tsukuba, Japan) and cultured in a chemically defined medium, SFM 101 (Nissui, Tokyo, Japan), supplemented with $1 \%$ fetal bovine serum (FBS), $100 \mathrm{U} / \mathrm{ml}$ penicillin $\mathrm{G}, 100 \mu \mathrm{g} / \mathrm{ml}$ streptomycin, and $10 \mathrm{ng} / \mathrm{ml}$ murine epidermal growth factor (mEGF) in a humidified atmosphere of $5 \% \mathrm{CO}_{2}$ in air at the optimal temperature of $33^{\circ} \mathrm{C}$. Cells wereseeded at $8.75 \times 10^{4} \mathrm{cells} / \mathrm{ml}$ in $12.5 \mathrm{~cm}^{2}$ flasks with a filter cap (Falcon \#353107; Corning Inc, Corning, NY, USA) and cultured in a normal $1 \mathrm{G}$ environment for $\sim 8$ hours to promote adherence. The flasks for cells cultured under microgravity (group CL) were filled completely with $\sim 47 \mathrm{ml}$ of medium, avoiding the introduction of air bubbles to diminish turbulence and shear forces, before loading on the device. The cells

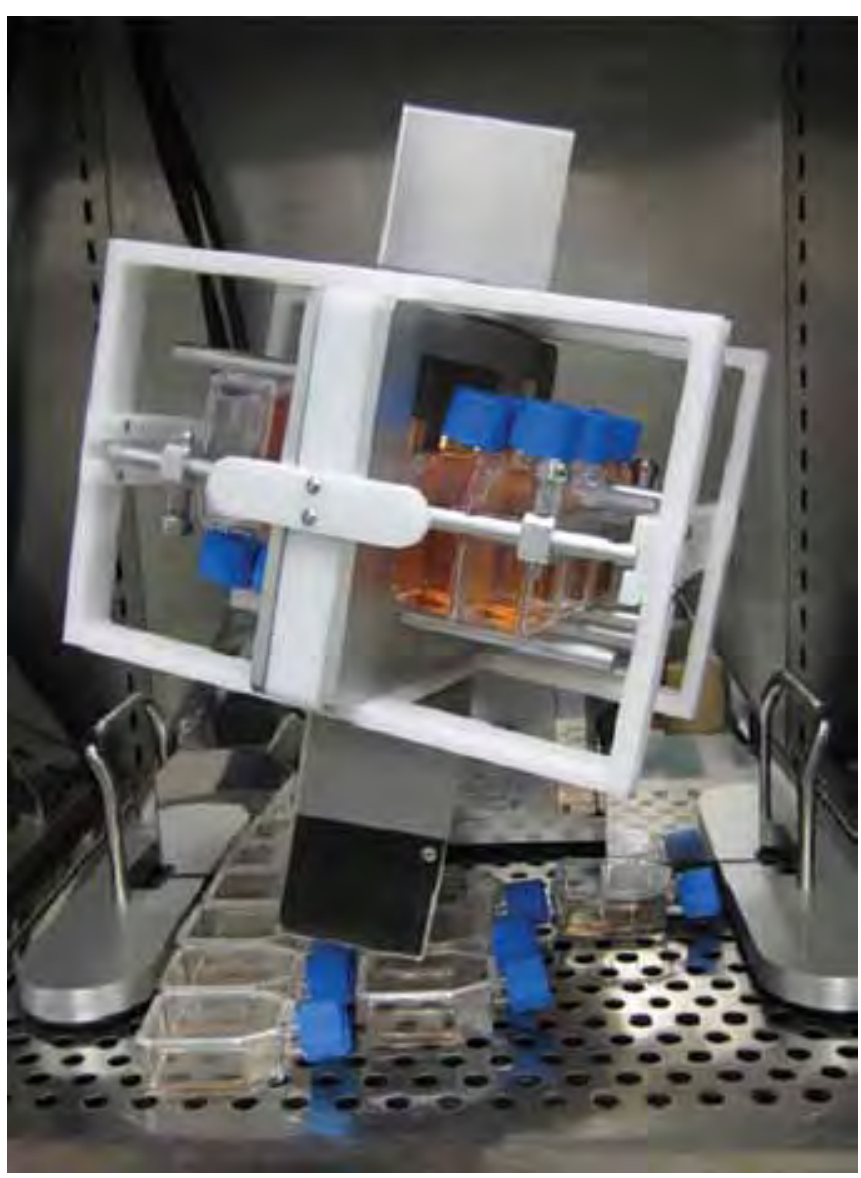

Fig. 1: Photograph of the Gravite: a 3D clinostat. The device is placed in a normal $\mathrm{CO}_{2}$ incubator. Culture flasks for cells cultured under microgravity, filled with medium, were placed in the center of the clinostat, and, those for the control group, with optimal amount of medium, cultured under normal gravity, were placed at the bottom shelf of the same incubator beneath the device

in a normal $1 \mathrm{G}$ environment (group C) were cultured in an average of $3 \mathrm{ml}$ of medium. Cells were cultured continuously for 10 days with sampling on days 0,3 , 5, 7 and 10; three samples were taken from each group at each timepoint, and cell counts were recorded for each flask. The device was stopped and brought to $1 \mathrm{G}$ temporarily for sampling. A preliminary experiment was performed to determine an initial cell count to avoid cultures reaching confluence before 10 days.

\section{Morphological Observations}

Morphological changes were examined using an inverted phase-contrast microscope (Eclipse TE 300; Nikon Co, Tokyo, Japan); images were captured at random intervals.

\section{Cell Counts}

Trypsin-EDTA (0.05\% trypsin, $0.02 \%$ EDTA, in Hank's Balanced Salt Solution) (SAFC Bioscience, KS, USA) was used to detach cells from the flasks. Cell counts were estimated within a standard microscope field of $5.0 \times 7.5 \mathrm{~mm}$ using a hemocytometer. Trypan blue $(0.4 \%)$ staining was used to exclude dead cells during counting. 


\section{RNA Isolation and Analysis}

Samples were collected using ISOGEN (Nippon Gene Co Ltd., Toyama, Japan) and total RNA was isolated according to the supplied protocol. Complementary DNA (cDNA) was synthesized from total RNA using the high capacity RNA-to-cDNA kit (Applied Biosystems, USA) according to the manufacturer's instructions. The reaction mixture was incubated at $37^{\circ} \mathrm{C}$ for 60 minutes, heated at $95^{\circ} \mathrm{C}$ for 5 minutes, and subsequently cooled to $4^{\circ} \mathrm{C}$. Using cDNA as the template, a real-time polymerase chain reaction was performed in 96-well plates (Applied Biosystems, USA) with thermal cycling parameters of $50^{\circ} \mathrm{C}$ for 2 minutes, $95^{\circ} \mathrm{C}$ for 10 minutes, and 40 cycles of $95^{\circ} \mathrm{C}$ for 15 seconds and $60^{\circ} \mathrm{C}$ for 1 minutes, using the TaqMan Universal PCR Master Mix (Applied Biosystems, USA) on an ABI Prism 7300 (Applied Biosystems, USA) to amplify the krt5 (Mm01305291_g1), krt13 (Mm00495199_m1) and involucrin (Mm00515219_s1) genes as proliferation or differentiation markers, with 18S ribosomal RNA (Hs99999901_s1) as the endogenous control.

\section{Western Blotting}

Gingival epithelial cells were lysed in RIPA buffer (1\% Triton X-100, 2 mM EDTA, $50 \mathrm{mM}$ Tris-HCl, $150 \mathrm{mM}$ $\mathrm{NaCl}, 1 \%$ sodium deoxycholate and $0.1 \%$ SDS) containing protease inhibitors (Complete Protease Inhibitor Cocktail; Roche, Mannheim, Germany). The samples were electrophoresed on SDS-polyacrylamide gels and transferred to polyvinylidene difluoride membranes. Nonspecific binding was blocked by immersing the membranes in protein-free blocking buffer (EzBlock Chemi; ATTO Corp, Tokyo, Japan) for 1 hour at room temperature. Then, the membranes were incubated with primary antibody (keratin 13 Mouse anti-human monoclonal antibody; LifeSpan BioSciences, Inc, Seattle, USA) followed by horseradish peroxidase-conjugated goat anti-mouse IgG secondary antibody (BD Biosciences, San Jose, USA). After washing, immunoreactive proteins were visualized on the membranes using enhanced chemiluminescence (Amersham, GE Healthcare, Cambridge, UK) and analyzed with a luminescent image analyzer (LAS-3000 Mini; Fujifilm, Tokyo, Japan).

\section{DATA ANALYSIS}

GraphPad Prism 5.0 for Mac OS X (GraphPad Software Inc, San Diego, CA, USA) was used for computation and statistical analyses. Differences between the mean cell numbers were tested for significance using student's t-test. Differences between group mean values $(n=9$; three replicates of three groups) were subjected to one-way analysis of variance (ANOVA) at $p<0.05$. After a significant difference was determined by ANOVA, Tukey's multiple range test was used as the post-hoc test at a $5 \%$ level of significance.

\section{RESULTS}

\section{Influence of Simulated Microgravity on Morphology}

Morphological differences were evident: cells in the group $C$ were flat with uniform surfaces, while the cells in the group CL showed distinct cell boundaries (Fig. 2).

\section{Influence of Simulated Microgravity on Cell Proliferation}

The mean number of cells in group CL was about threefold greater than that in group $C$ on day $10(p<0.05)$. However, cell growth was not significantly different between the two groups up to day 7 (Graph 1).

\section{Influence of Simulated Microgravity on Proliferation and Differentiation Marker Expression}

The expression of proliferation and differentiation markers was significantly different between the groups. The expression level of the proliferation marker krt5 was significantly greater $(\mathrm{p}<0.05,1.8$-fold) in group $\mathrm{CL}$ than in group $C$ on day 10 (Graph 2A). The expression levels of the differentiation marker krt13 were significantly greater ( $p<0.05,7.0$ - and 12.7-fold respectively) in group CL on days 7 and 10 than in group C (Graph 2B). The expression level of the differentiation marker involucrin was significantly greater in group $\mathrm{CL}(\mathrm{p}<0.05$, 2.7 -fold) than in group $C$ on day 7 . However, the difference was not significant between the two groups on day 10 (Graph 2C).

\section{Stimulation of Gingival Differentiation by Simulated Microgravity}

Increased krt13 protein expression was seen in group CL compared to group C on day 10 by western blotting (Fig. 3).

\section{DISCUSSION}

Microgravity is believed to have various effects on animal cells. We are interested in preserving a healthy periodontal or peri-implant state under microgravity conditions. Several studies on osteoblasts exposed to microgravity using a $3 \mathrm{D}$ clinostat have been performed. ${ }^{15,16}$ The results of all indicated that long-term exposure to microgravity inhibits osteoblast differentiation. However, no studies have examined soft-tissue cells under microgravity conditions using a 3D clinostat. Therefore, our 


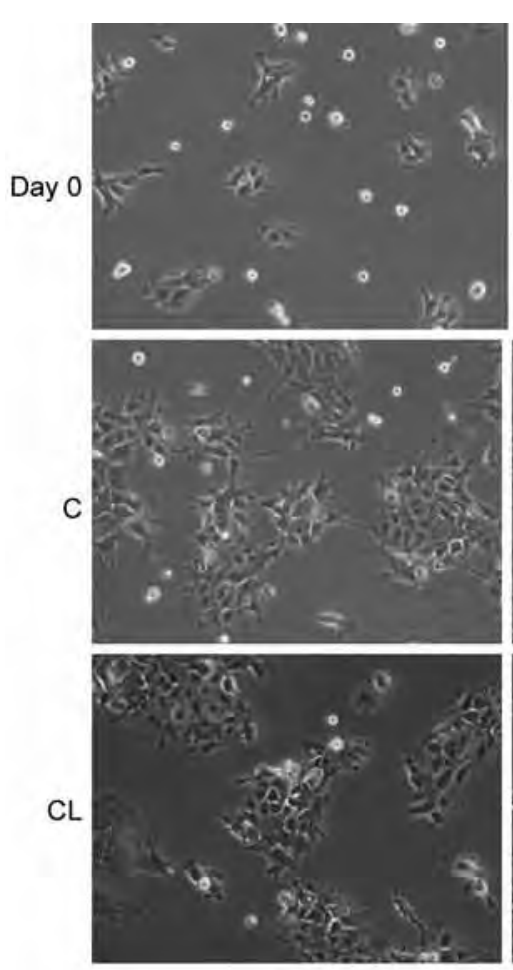

Day 3
Seeded cells: $8.75 \times 10^{4}$ cells $/ \mathrm{m}$

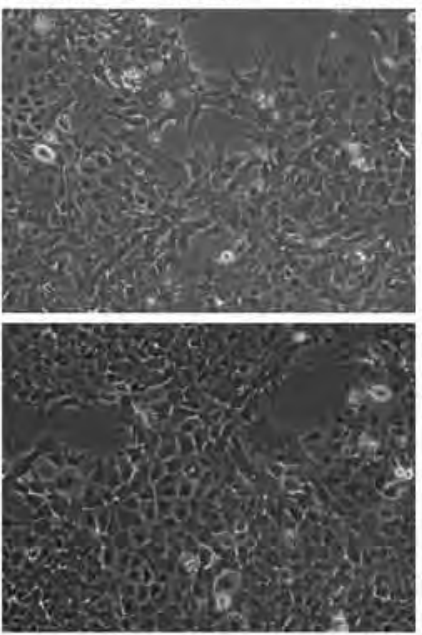

Day 5

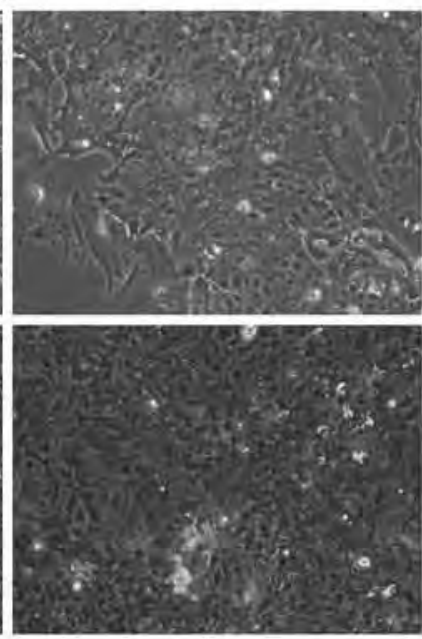

Day 7

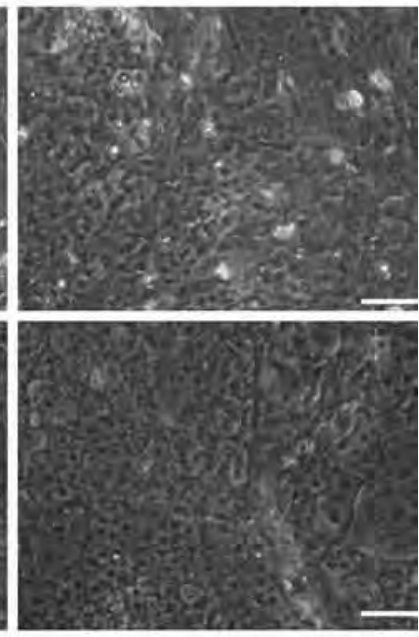

Day 10

Scale bar: $100 \mu \mathrm{m}$

Fig. 2: Influence of simulated microgravity on cell morphology. Cells were seeded at $8.75 \times 10^{4}$ cells $/ \mathrm{ml}$, and cell morphology was observed over time. Morphological differences were evident; the group $\mathrm{C}$ cells were flat with uniform surfaces, whereas the group $\mathrm{CL}$ cells showed distinct cell boundaries $(\mathrm{bar}=100 \mu \mathrm{m})$

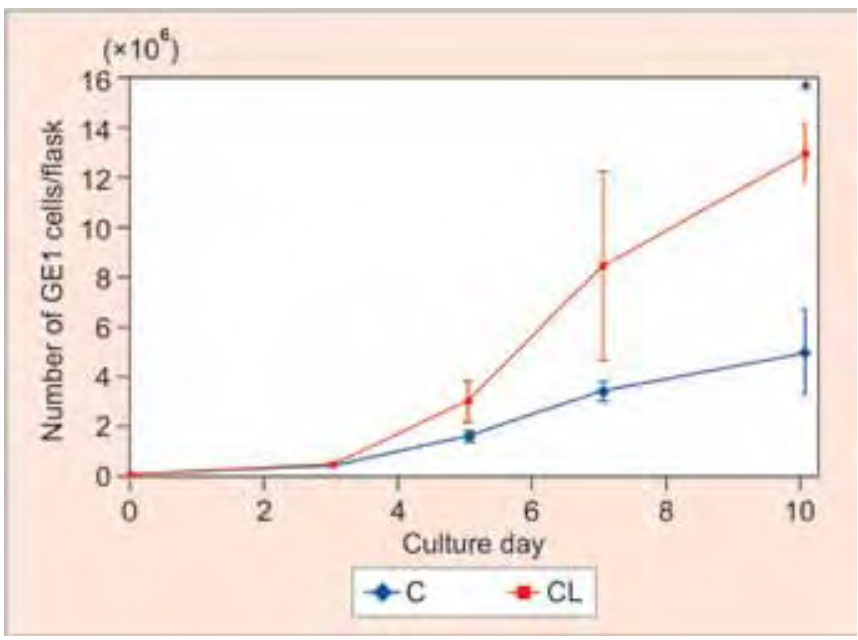

Graph 1: Influence of simulated microgravity on cell proliferation. Cells were cultured continuously for 10 days and sampled on days $0,3,5,7$, and 10 when cell counts were recorded for each flask. The cell proliferation rate was significantly (three-fold) higher in group $C L$ than in group $C\left(^{*}: p<0.05\right)$

aim was to examine the impact of microgravity on soft tissue cells. In this study, we investigated the influence of simulated microgravity on GE1 cells.

In earlier cell culture experiments on rat bone marrow stromal cells in a 3D clinostat, ${ }^{17}$ morphological changes were detected; compared to the control, cells in the $3 \mathrm{D}$ clinostat became more dome-shaped and smaller in size. In the present study, we did not evaluate the 3D structures of cells, as changes in morphology were not relevant; however, morphological changes were evident.

The ability of cells to sense mechanical signals from the environment requires that either mechanoreceptors are in direct contact with the extracellular space, or that a mechanoreceptor can detect changes though a physical intermediary, such as pressure or fluid shear on the plasma membrane. Candidate mechanoreceptors span the gamut of specific receptors for force that respond by inducing conformational changes in proteins: candidate structures, such as primary cilium, or harder-to-define responders that regulate protein interactions, such as mechanically induced alterations in components of plasma lipid, or interactions with the extracellular matrix (ECM) or the cytoskeleton. The cytoskeleton regulates cell-to-cell connections, and may enable cells to sense the mechanical environment. ${ }^{18}$ From our observations of morphological change, we surmised that microgravity might have an impact on the cytoskeleton, or the cell-to-cell connections, of GE1 cells. Further analysis is required to identify the mechanism involved in this phenomenon.

Gingival epithelial cells were originally established to demonstrate stable infinite growth and morphological differentiation as a 3D stratifying oral epithelium, without any feeder layer, over $\sim 2$ weeks of cell culture without passage. ${ }^{19}$ The GE1 cell line has a sulcular epithelium 

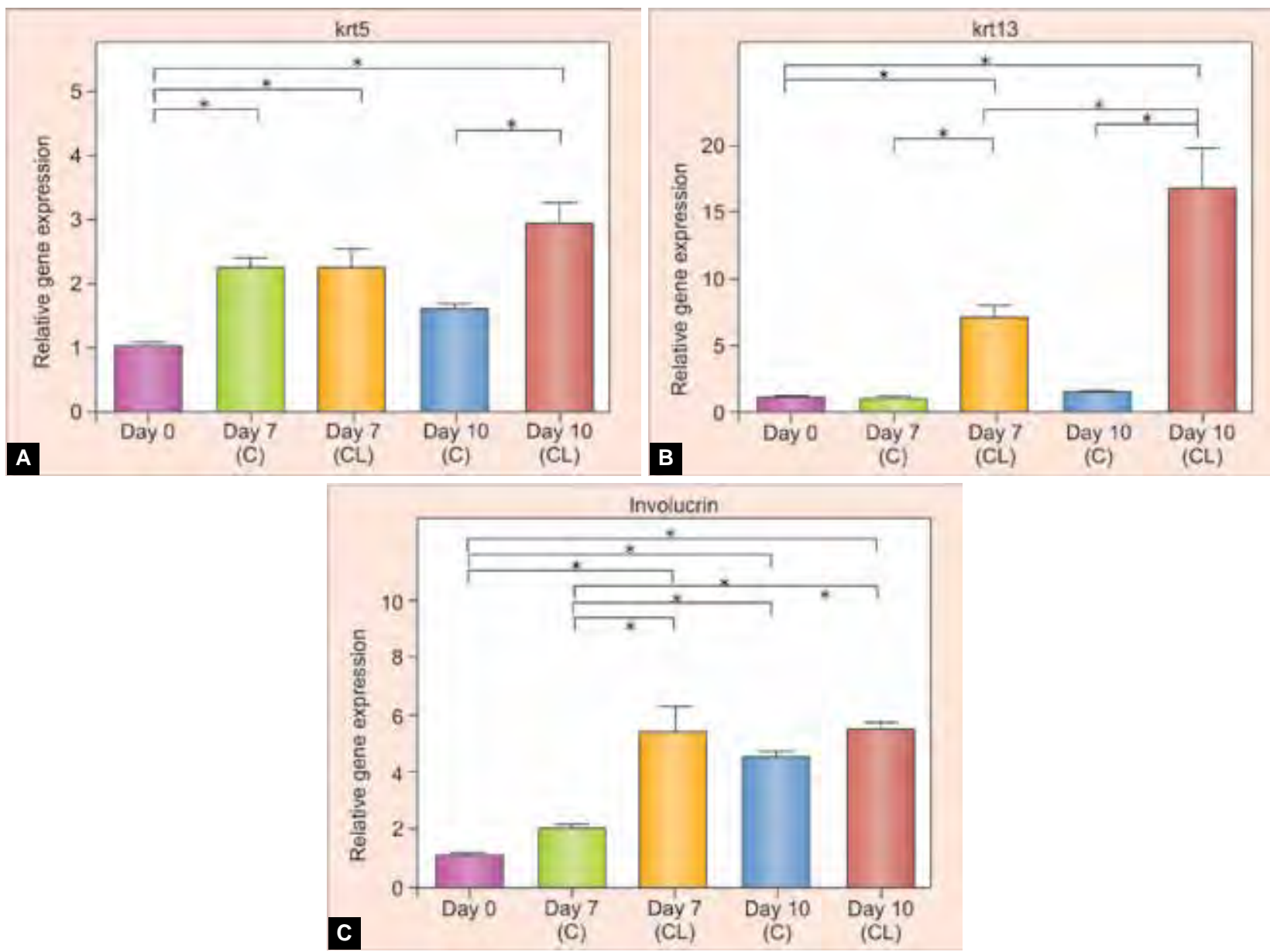

Graphs 2A to C: Influence of simulated microgravity on the expression of cell proliferation and differentiation markers. Real-time polymerase chain reaction was performed to amplify the krt5 (A), krt13 (B) and involucrin (C) genes

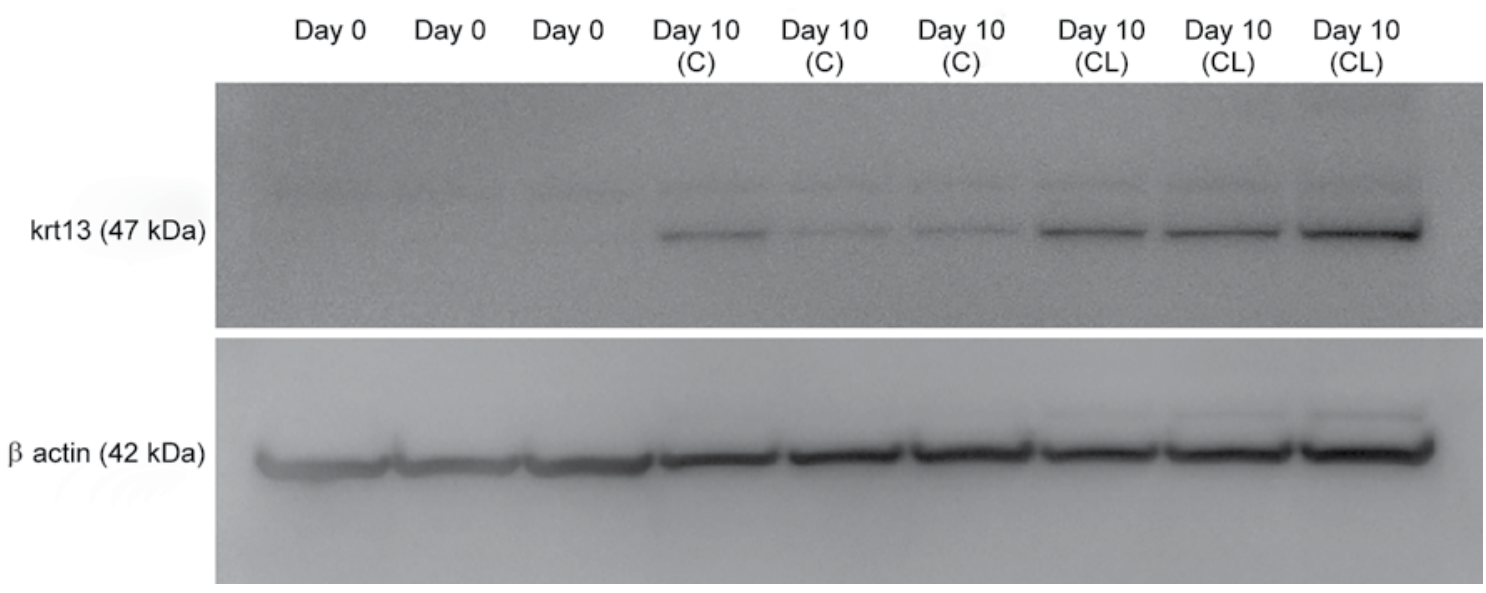

Fig. 3: Influence of simulated microgravity on krt13 production. Whole cell lysates were prepared, and expression was examined by western blotting. The experiment was performed three times, and similar results were obtained

phenotype insubconfluent culture, whereas the culture phenotype becomes similar to that of keratinized oral gingival epithelium when the cells are confluent and multilayered. One of the merits of GE1 cells is their expression of different phenotypes of stratified epithelium within one cell culture as they proliferate or differentiate. Gingival epithelial cells are adherent and must be cultured in an appropriate environment. The 3D clinostat used in this study is considered an optimal instrument because the loading volume allows testing of multiple samples under identical conditions simultaneously, samples can be cultured in a commonly available container, and the system can be kept in a standard controllable $\mathrm{CO}_{2}$ incubator.

Previous reports on human mesenchymal stem cells demonstrated that microgravity generated by a 3D clinostat accelerates cell proliferation, ${ }^{20}$ as was observed for GE1 cells in the present study. Epithelial tissues express 
a different keratin pair depending on the cell type; for example, all stratified squamous epithelial cells express $\mathrm{krt5} / \mathrm{krt14.} .^{21}$ The krt5/krt14 pair is normally expressed in the basal layer of stratified epithelial cells, and its expression decreases as these cells differentiate and lose their proliferation potential. ${ }^{22}$ Although proliferation marker expression levels were not examined on days 3 and 5 , and the culture was conducted such that the cells would not reach confluence during the experiment, expression of the proliferation marker krt5 was maintained at a high level on day 10 in group CL compared to group C. This result may have occurred because the microgravity environment helped to preserve cell proliferation.

Krt13 is a common differentiation marker that is expressed with krt4 in the differentiating supra-basal cells of the oral epithelium. ${ }^{23-25}$ Involucrin is a useful marker for the early stages of the terminal differentiation pathway. Involucrin is synthesized immediately after keratinocytes have left the basal layer and started to enlarge, but before the onset of envelope cross-linking, which occurs only in the outermost layers. ${ }^{26}$ In the present study, the gene expressions of krt13 and involucrin, which are markers of more differentiated stratified epithelia, and also, the protein production of krt13, increased compared to those in cells under normal $1 \mathrm{G}$ condition. This result may have occurred because the cells exposed to microgravity were stimulated to proliferate faster and, as a result, their population expanded sufficiently to express the differentiation markers earlier than cells under a normal $1 \mathrm{G}$ condition.

This study was designed to characterize the impact of microgravity on GE1 cells using a 3D clinostat. However, it has several limitations. The sample containers in the $3 \mathrm{D}$ clinostat rotate in an incubator. Therefore, purchasable containers available for use were limited; thus, the number of obtainable samples was downsized, and we were limited to detecting changes in the expression of certain genes over time. A greater depth of information could have been obtained by analyzing more genes or repeating trials focusing on different factors, such as protein production, cytoskeletal structure, or cell-to-cell attachment. We could have tried using the clinostat on cells after they became confluent to determine the effect of microgravity on GE1 cell differentiation. Nevertheless, we detected changes in gene expression by exposing GE1 cells to microgravity and have provided some ideas for future studies.

Today, astronauts are living and working in space aboard the International Space Station (ISS); moreover, in the near future, it may become common for people to go back and forth between earth and space. However, the mechanisms of major physical stress factors that may affect human health are not yet fully understood. One of these factors is microgravity, exposure to which can affect almostall human physiological systems, including musculoskeletal, neurovestibular, cardiovascular, immune, and endocrine functions. ${ }^{3}$ Therefore, microgravity may also affect systems in dental areas; therefore, studies in this field are warranted.

In dentistry, tissue engineering is considered to be a new frontier in the regeneration of missing oral tissues/ organs. ${ }^{27,28}$ Our results suggest that the simulated microgravity environment stimulates GE1 cell proliferation and differentiation. These findings could promote the use of a microgravity environment to regenerate gingival cells.

\section{CONCLUSION}

A simulated microgravity environment accelerated GE1 cell proliferation and differentiation. In addition, the simulated microgravity environment may have influenced cell-to-cell contact or the cytoskeleton of GE1 cells. These findings indicate that the microgravity environment may affect peri-implant junctional epithelial attachment and gingival attachment during space flight.

\section{ACKNOWLEDGMENTS}

This study was supported by a Grant-in-Aid for Challenging Exploratory Research (no. 24659865) from the Ministry of Education, Culture, Sports, Science and Technology of Japan. We are grateful to Dr Louis Yuge of the Graduate School of Health Sciences, Hiroshima University, Japan, and to Drs Yumi Kawahara and Takahiro Fukazawa of Space Bio-Laboratories Co, Ltd., Hiroshima, Japan, for helping with the experiment.

\section{REFERENCES}

1. Graebe A, Schuck EL, Lensing P, Putcha L, Derendorf H. Physiological, pharmacokinetic, and pharmacodynamic changes in space. J Clin Pharmacol 2004;44(8):837-853.

2. Newman DJ, Jackson DK, Bloomberg JJ. Altered astronaut lower limb and mass center kinematics in downward jumping following space flight. Exp Brain Res 1997;117(1):30-42.

3. Zayzafoon M, Meyers VE, McDonald JM. Microgravity: the immune response and bone. Immunol Rev 2005;208:267-280.

4. Crawford-Young SJ. Effects of microgravity on cell cytoskeleton and embryogenesis. Int J Dev Biol 2006;50(2-3):183-191.

5. Hughes-Fulford M, Lewis Ml. Effects of microgravity on osteoblast growth activation. Exp Cell Res 1996;224(1):103-109.

6. Tairbekov MG, Margolis LB, Baibakov BA, Gabova AV, Dergacheva GB. Cell growth and motility in culture (in vitro) under microgravity conditions. The Fibroblast Experiment. Izv Akad Nauk Ser Biol 1994 Sep-Oct;5(5):745-750.

7. Saxena R, Pan G, Dohm ED, McDonald JM. Modeled microgravity and hindlimb unloading sensitize osteoclast precursors to RANKL-mediated osteoclastogenesis. J Bone Miner Metab 2011;29(1):111-122. 
8. Barzegari A, Saei AA. An update to space biomedical research: tissue engineering in microgravity bioreactors. Bioimpacts 2012;2(1):23-32.

9. Berglundh T, Lindhe J, Ericsson I, Marinello CP, Liljenberg B, Thomsen P. The soft tissue barrier at implants and teeth. Clin Oral Implants Res 1991;2(2):81-90.

10. Comut AA, Weber HP, Shortkroff S, Cui FZ, Spector M. Connective tissue orientation around dental implants in a canine model. Clin Oral Implants Res 2001;12(5):433-440.

11. Brito C, Tenenbaum HC, Wong BK, Schmitt C, NogueiraFilho $\mathrm{G}$. Is keratinized mucosa indispensable to maintain peri-implant health? A systematic review of the literature. J Biomed Mater Res B Appl Biomater 2014;102(3):643-650.

12. Yeung SC. Biological basis for soft-tissue management in implant dentistry. Aust Dent J 2008;53(1):S39-42.

13. Miyata K, Takebe J. Anodized-hydrothermally treated titanium with a nanotopographic surface structure regulates integrin$\alpha 6 \beta 4$ and laminin-5 gene expression in adherent murine gingival epithelial cells. J Prosthodont Res 2013;57(2):99-108.

14. Chai WL, BrookIM,Palmquist A, van NoortR, MoharamzadehK. The biological seal of the implant-soft tissue interface evaluated in a tissue engineered oral mucosal model. J R Soc Interface 2012;9(77):3528-3538.

15. Yuge L, Hide I, Kumagai T, Kumei Y, Takeda S, Kanno M, Sugiyama M, Kataoka K. Cell differentiation and p38(MAPK) cascade are inhibited in human osteoblasts cultured in a three-dimensional clinostat. In Vitro Cell Dev Biol Anim 2003; 39(1-2):89-97.

16. Makihira S, Kawahara Y, Yuge L, Mine Y, Nikawa H. Impact of the microgravity environment in a 3-dimensional clinostat on osteoblast- and osteoclast-like cells. Cell Biol Int 2008;32(9):1176-1181.

17. Mitsuhara T, Takeda M, YamaguchiS, Manabe T, Matsumoto M, Kawahara Y, Yuge L, Kurisu K. Simulated microgravity facilitates cell migration and neuroprotection after bone marrow stromal cell transplantation in spinal cord injury. Stem Cell Res Ther 2013;4(2):35.

18. Thompson WR, Rubin CT, Rubin J. Mechanical regulation of signaling pathways in bone. Gene 2012;503(2):179-193.

19. Hatakeyama S, Ohara-Nemoto Y, Yanai N, Obinata M, Hayashi $\mathrm{S}$, Satoh M. Establishment of gingival epithelial cell lines from transgenic mice harboring temperature sensitive simian virus 40 large T-antigen gene. J Oral Pathol Med 2001;30(5):296-304.

20. Yuge L, Kajiume T, Tahara H, Kawahara Y, Umeda C, Yoshimoto R, Wu S, Yamaoka K, Asashima M, Kataoka K, Ide T. Microgravity potentiates stem cell proliferation while sustaining the capability of differentiation. Stem Cells Dev 2006;15(6):921-929.

21. Nelson WG, Sun TT. The 50- and 58-kdalton keratin classes as molecular markers for stratified squamous epithelia: cell culture studies. J Cell Biol 1983;97(1):244-251.

22. Alam H, Sehgal L, Kundu ST, Dalal SN, Vaidya MM. Novel function of keratins 5 and 14 in proliferation and differentiation of stratified epithelial cells. Mol Biol Cell 2011;22(21):4068-4078.

23. Bragulla HH, Homberger DG. Structure and functions of keratin proteins in simple, stratified, keratinized and cornified epithelia. J Anat 2009;214(4):516-559.

24. Moll R, Divo M, Langbein L. The human keratins: biology and pathology. Histochem Cell Biol 2008;129(6):705-733.

25. Hatakeyama S, Hayashi S, Yoshida Y, Otsubo A, Yoshimoto K, Oikawa Y, Satoh M. Retinoic acid disintegrated desmosomes and hemidesmosomes in stratified oral keratinocytes. J Oral Pathol Med 2004;33(10):622-628.

26. Watt FM. Involucrin and Other Markers of Keratinocyte Terminal Differentiation. J Invest Dermatol 1983;81(1):100s-103s.

27. Egusa H, Sonoyama W, Nishimura M, Atsuta I, Akiyama K. Stem cells in dentistry-Part I: Stem cell sources. J Prosthodont Res 2012;56(3):151-165.

28. Koyano,K. Toward a new era in prosthdontic medicine. J Prosthodont Res 2012;56(1):1-2. 